\title{
Characterization of Sub-Bandgap Plasmon Excitations in Transparent Conducting Oxides with Electron Energy-Loss Spectroscopy
}

\author{
Julia I. Deitz ${ }^{1}$, John M. Walls ${ }^{2}$, Jake W. Bowers ${ }^{3}$, Tyler J. Grassman ${ }^{3,4}$, and David W. McComb ${ }^{1,4}$ \\ 1. Center for Electron Microscopy and Analysis, Department of Materials Science and Engineering, The \\ Ohio State University, Columbus, OH, United States. \\ 2. Centre for Renewable Energy Systems Technology, Dept. of Mechanical, Electrical and \\ Manufacturing Engineering, Loughborough University, Loughborough, Leicestershire, UK. \\ ${ }^{3 .}$ Dept. of Electrical \& Computer Engineering, The Ohio State University, Columbus, OH, USA. \\ 4. Dept. of Materials Science \& Engineering, The Ohio State University, Columbus, OH, USA. \\ * Corresponding author: deitz.13@osu.edu
}

Transparent conducting oxides (TCOs) are a critical component in a wide range of optoelectronic technologies, including nearly all thin-film photovoltaics (PV) technologies as front-side and/or interfacial electrodes. However, TCOs are known to exhibit collective free carrier excitation (plasmon) resonances at energies below their semiconducting bandgaps, typically in the near infrared (NIR) spectral region. These lead to wide absorption bands centered around the resonant frequencies, reducing the requisite transparency which is detrimental to overall PV performance [1]. Characterizing uniformity of these resonances, how they change in proximity to surfaces and interfaces, whether and how they can be controlled via intentional compositional manipulation could lead to vital information to mitigate such effects. Scanning transmission electron microscopy (STEM) based electron energy-loss spectroscopy (EELS) can provide such information in a spatially-resolved manner, but due to past challenges associated with accessible energy resolution, few investigations of these low-energy plasmons via STEM-EELS have been reported. With advances in monochromation for STEM-EELS, this information is now much more accessible [2]. In this contribution, we will present results from STEM-EELS characterization of NIR subbandgap plasmons in multiple commonly used TCOs.

We have investigated the plasmonic signals via low-loss STEM-EELS, performed in a monochromated FEI Titan ${ }^{3}$ 60-300 microscope at $60 \mathrm{kV}$, in three different TCO materials commonly used in the fabrication of solar cells and other optoelectronic devices: In-doped $\mathrm{SnO}_{2}$ (ITO), F-doped $\mathrm{SnO}_{2}$ (FTO), and Al-doped $\mathrm{ZnO}$ (AZO). Using Hall-based carrier concentration measurements and optical absorption spectroscopy, plasmon resonances in these materials were determined to reside (peak) at $0.98 \mathrm{eV}, 0.76 \mathrm{eV}$, and $0.49 \mathrm{eV}$, respectively. STEM-EELS analysis reveals distinct inelastic signals at $0.49 \mathrm{eV}$ and $0.71 \mathrm{eV}$ in the AZO and FTO materials, respectively, consistent with the external measurements; an example low-loss spectrum from AZO is shown in Figure 1.

In the FTO sample, an additional, more intense peak at $0.50 \mathrm{eV}$ is also observed, suggesting that another sub-bandgap feature that may not be optically active is also present. Spatial mapping of plasmonic resonances at an $\mathrm{AZO} / \mathrm{ZnO}$ interface in a solar cell device was analyzed with low-loss STEM-EELS, as shown in Figure 2. It can be seen that the low-loss $0.5 \mathrm{eV}$ signal only exists in the doped $\mathrm{ZnO}$ region where a high free carrier density will reside. Surprisingly, the ITO sample did not exhibit any sub-bandgap features. The reason for this is still under investigation, but it may be that this feature lies below our present STEM-EELS detection limit (the ITO exhibited a lower plasmonic absorption strength than the other TCOs in this study). 


\section{References:}

[1] J Gwamuri et al., Mater. Renew. Sustain. Energy 4 (2015), p. 12.

[2] CS Granerød et al., Phys. Rev. B 98, (2018), p. 115301.

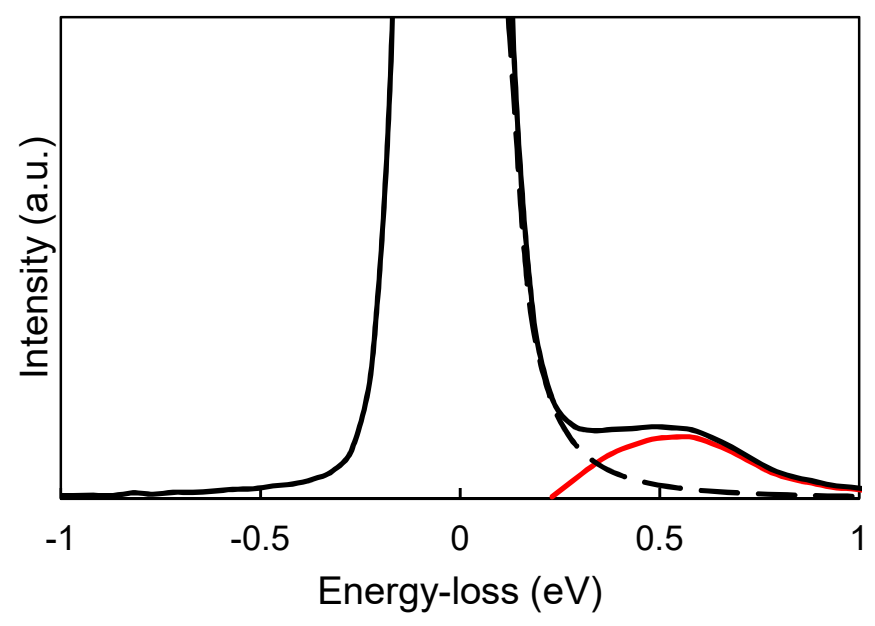

Figure 1. EELS spectrum on Al doped $\mathrm{ZnO}$ sample. The solid black line represents the raw EELS spectrum, the dotted black line represents the fitted zero-loss peak, and the red line represents the remaining inelastic signal.
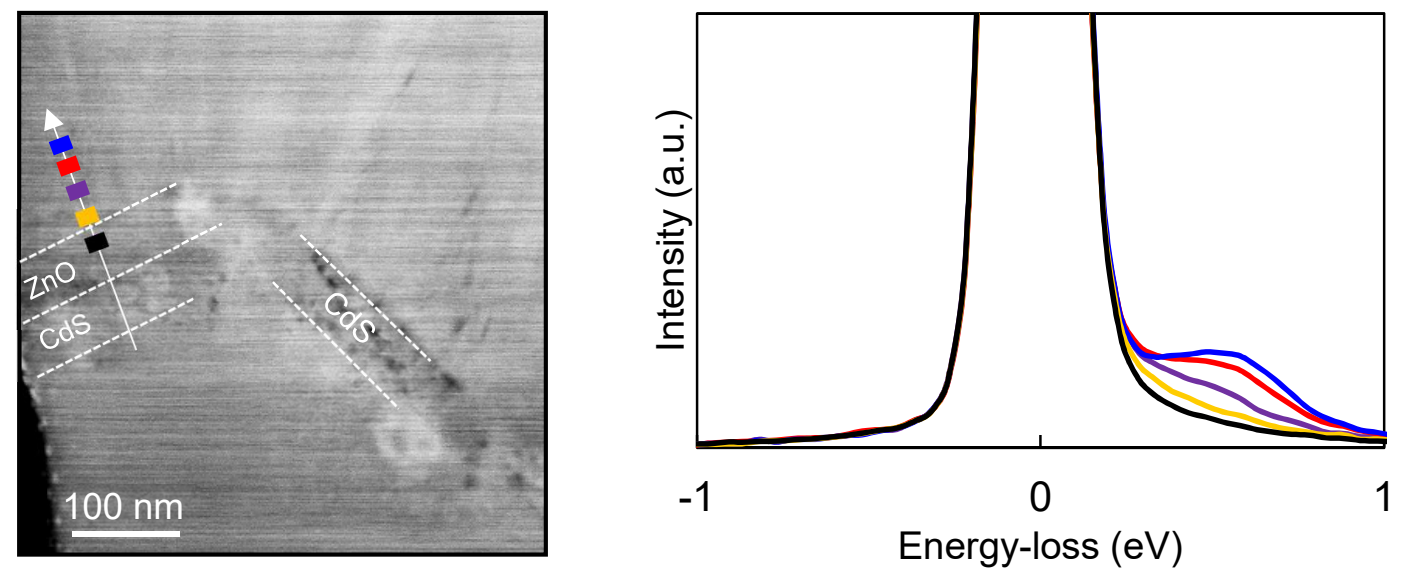

Figure 2. Image of EELS line scan going from a pure $\mathrm{ZnO}$ region to $\mathrm{Al}$ doped $\mathrm{ZnO}$ (left) and associated EELS spectra with increasing $0.5 \mathrm{eV}$ inelastic signal with increased doping (right). Zero-loss peaks were normalized to account for thickness variations. 